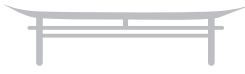

\title{
What Do J Need to Know to Read This Book?
}

We hope this book can be read in three ways: as an art book that delights simply by the perusal of it, as a history book that provides a little insight into an aspect of Japanese culture rarely mentioned in standard surveys, and finally as a problem book that provides challenging exercises at both the high school and college levels.

Readers who intend to tackle the problems may wish to know at the outset the prerequisites. One requirement looms above all: patience. On first encounter with a sangaku problem there is a considerable "choke" factor. At first glance, Western students will find many of the problems strange, unlike those they have seen before, and one's first reaction tends to be, "I can't do that!"

Do not despair! Half the problems in Sacred Mathematics require no more than the most elementary methods, taught in any high school geometry course. The individual steps are no larger than in typical textbook problems. What is different is that sangaku problems are frequently far more intricate than the usual exercises American students encounter. Instead of running four or five lines, proofs may run four or five pages-if not ten. What is more, it is necessary to bring to bear everything you've learned from your geometry course. Sooner or later you will require virtually every theorem about circles, quadrilaterals, triangles, and tangents that you have proved. Some of the more difficult exercises require a good deal more than that. You should not be surprised if you spend hours-or days-working the advanced problems.

Following patience, a number of specific tools are required to solve sangaku problems. Because Sacred Mathematics is not intended to be a textbook, however, for the most part we do not teach basic methods. If you do try the problems it is a good idea to have a standard geometry text handy as a reference. A few suggestions, ranging from the elementary to the advanced are offered in "For Further Reading" on page 337; nevertheless, by way of helpful hints we can be a bit more specific here about what you will need. 
A good drawing is indispensable. Many of the problems are fairly subtle, and it is not enough to make a rough sketch, which will deceive you; you need to make an accurate drawing that reflects the true conditions of the problem. Often the route to a solution becomes obvious when you've drawn in the appropriate auxiliary lines.

The single most important mathematical tool will be the Pythagorean theorem. This basic theorem, which was known to the Japanese through China, is used constantly throughout, and a large percentage of the problems can be solved with nothing more. If you are uncomfortable with the Pythagorean theorem, the problems in this book will be extremely difficult.

Hand in hand with the Pythagorean theorem, many, if not most, of the problems require solving quadratic equations. Not only will you frequently need the quadratic formula, which was also known to the Japanese, but it will often prove more convenient to solve the equation by "completing the square." Nearly as often you will encounter quadratics in the square root of a quantity, usually the radius of a circle, $\sqrt{r}$, and so you will need to know the basic methods for handling square roots, such as rationalizing denominators.

After the Pythagorean theorem and quadratics, the most frequently needed tool is probably properties of similar triangles, those theorems that go by names like AAA and SAS. You need all of them. Likewise, you will require virtually all the trigonometric identities involving sine, cosine, and tangent, that is, not only the basic Pythagorean identity, $\sin ^{2} \theta+\cos ^{2} \theta=1$ but every variation on it, as well as all the half-angle and double-angle formulas.

The law of cosines also crops up often. This is the generalization of the Pythagorean theorem for nonright triangles that gives the third side of a triangle $c$ in terms of the other two sides: $c^{2}=a^{2}+b^{2}-2 a b \cos \theta$, where $\theta$ is the included angle between sides $a$ and $b$. The law of sines also figures occasionally: $a / \sin A=b / \sin B=c / \sin C$, for triangles with vertex angles $A, B$, $C$ and opposite sides $a, b, c$. Although traditional Japanese mathematicians did not explicitly use trigonometric functions, they did employ the equivalents and all these relationships were understood by them. Solutions to problems in chapters 4 and 5 will give a better idea of how those mathematicians operated.

Frequently, problems are solved by considering the area of triangles, rather than the lengths of the sides. In both this context and when employing the Pythagorean theorem, one basic fact crops up repeatedly: that two 
tangent segments to a circle drawn from an external point $P$ are equal in length. This is sometimes referred to as the "tangent segments theorem." If you have not seen this proven in an elementary course, you may wish to prove it now; it is very easy. The "intersecting chords theorem" is also frequently encountered: if two chords intersect in a circle, the product of the lengths of the segments of one chord equal the product of the lengths of the segments of the other chord (figure 0.1). Other theorems we introduce as needed.

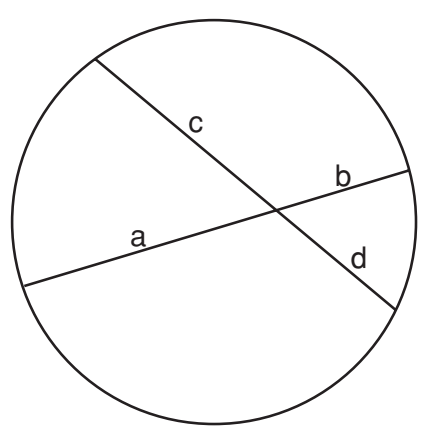

Figure 0.1. The intersecting chords theorem: $a \times b=c \times d$

For solving some of the more advanced problems, a "modern" technique that proves extremely useful is that of "inversion." However, because inversion is not generally taught in schools anymore, we include a chapter (10) to explain this powerful method.

A handful of problems requires calculus, but except for a very small number, this does not go beyond basic differentiation and integration. 
